\title{
Outpatient Craniotomy for Brain Tumor: A Pilot Feasibility Study in 46 Patients
}

\author{
Mark Bernstein
}

\begin{abstract}
Background: Since 1991 the author has routinely performed awake craniotomy for intraaxial brain tumors with low complication rate and low resource utilization. In late 1996 a pilot study was initiated to assess the feasibility of performing craniotomy for tumor resection as an outpatient procedure. Methods: A rigorous protocol was developed and adhered to, based around the patient's arrival at hospital at 6:00 a.m, undergoing image-guided awake craniotomy with cortical mapping, and being discharged by 6:00 p.m. Results: During the 48 month period from December 1996 to December 2000, 245 awake craniotomies were performed and of those, 46 patients were entered into the outpatient craniotomy protocol. Pathology in the 46 intent-to-treat group was: 21 metastasis, 19 glioma, and six miscellaneous. Four patients required conversion to inpatients and one patient was readmitted later the same evening due to headache. Thus $41 / 46$ patients successfully completed the protocol (89\%). There were five complications in the 46 intent-to-treat group (10.9\%). Conclusions: Outpatient craniotomy for brain tumor is a feasible option which appears safe and effective for selected patients. Besides being resource-friendly, the procedure may be psychologically less traumatic to patients than standard craniotomy for brain tumor. Proper prospective studies including satisfaction surveys would help resolve these issues and will be the next step.
\end{abstract}

RÉSUMÉ: Crâniotomie pour tumeur cérébrale en chirurgie ambulatoire: une étude pilote de faisabilité chez 46 patients. Introduction: Depuis 1991, l'auteur a effectué de routine l'exérèse de tumeurs cérébrales intra-axiales par crâniotomie sans anesthésie générale, en chirurgie ambulatoire. Le taux de complications est bas et l'utilisation des ressources est minime. En 1996, une étude pilote a été mise sur pied pour évaluer la faisabilité de la crâniotomie pour l'exérèse de tumeurs en chirurgie ambulatoire. Méthodes: Un protocole rigoureux a été développé et réalisé, à partir du moment où le patient arrive à l'hôpital à 6:00, subit une crâniotomie sans anesthésie générale guidée par imagerie avec cartographie corticale jusqu'au moment où il quitte l'hôpital à 18:00. Résultats: Pendant une période de 48 mois entre Décembre 1996 et décembre 2000, 245 crâniotomies sans anesthésie générale ont été effectuées dont 46 selon le protocole de chirurgie d'un jour. Les diagnostics suivants ont été posés à l'examen anatomopathologique chez les 46 patients inclus dans l'étude: 21 métastases, 19 gliomes et six tumeurs variées. Chez quatre patients on a dû recourir à l'hospitalisation et un patient a été réadmis le soir même pour céphalée. Donc 41 des 46 patients ont complété le protocole avec succès (89\%). Cinq complications sont survenues dans le groupe total des patients inclus dans l'étude (10.9\%). Conclusions: La crâniotomie en externe pour l'exérèse de tumeurs cérébrales est une option valable qui semble sûre et efficace chez des patients bien choisis. En plus d'économiser les ressources, cette approche peut être moins traumatique psychologiquement pour les patients par rapport à la crâniotomie standard pour une tumeur cérébrale. Des études prospectives bien structurées, incluant l'évaluation de la satisfaction, aideraient à éclairer ces aspects et constituent la prochaine étape à réaliser.

Can. J. Neurol. Sci. 2001; 28: 120-124

Over the last several decades there has been a trend toward shorter, less invasive surgical procedures within every surgical discipline. Examples include endoscopic bowel resection ${ }^{1}$ and laparoscopic cholecystectomy, ${ }^{2}$ arthroscopic joint surgery, ${ }^{3}$ transurethral endoscopic surgery for prostate and bladder disorders, ${ }^{4}$ and cardiac angioplasty. ${ }^{5}$ In the neurosurgical arena, the transsphenoidal approach revolutionized the efficacy and complications of pituitary surgery ${ }^{6}$ and further advances, with endoscopes replacing microscopes, have further minimised the procedure. ${ }^{7}$ Endoscopes have also allowed for minimally invasive sympathectomy, ${ }^{8}$ and various types of intracranial surgery ${ }^{9}$ and spinal surgery. ${ }^{10}$ Microscopic lumbar discectomy can reduce postoperative incisional pain and improve

From the Division of Neurosurgery, Toronto Western Hospital, University Health Network, and Department of Surgery, University of Toronto, Toronto, ON Canada ReCEIVEd OCTOBER 5, 2000. ACCEPTED IN FINAL FORM JANUARY 24, 2001. Reprint requests to: Mark Bernstein, Division of Neurosurgery, Toronto Western Hospital, Suite 2-405 McLaughlin Pavilion, 399 Bathurst Street, Toronto, Ontario M5T 2S8 Canada 
illumination for the surgeon working in a deep hole, and recent addition of the arthroscope has further limited the invasiveness of the procedure for selected patients. ${ }^{11}$ Intra-arterial therapy has facilitated dilatation of stenotic carotid arteries, ${ }^{12}$ coiling of aneurysms, ${ }^{13}$ and obliteration of arteriovenous malformations. ${ }^{14}$

Stereotactic frames fixed to the head have allowed for biopsy of deep lesions, ${ }^{15}$ functional neurosurgery for movement disorders and other conditions, ${ }^{16}$ placement of radiation seeds within tumors, ${ }^{17}$ and numerous other applications which require a very small hole in the scalp and skull. Ultimately, the novel application of stereotactic frames allowed for the development of stereotactic noninvasive radiation for brain lesions ${ }^{18}$ which is not strictly a surgical procedure, but essentially replaces one with an outpatient procedure. Frameless stereotactic navigation systems ${ }^{19}$ and intra-operative magnetic resonance imaging $(\mathrm{MRI})^{20}$ have been developed within the last decade for pinpoint placement of the surface projection of intracranial lesions to facilitate the planning of small scalp (which can often be linear) and bone flaps, and for accurate localisation of intracranial lesions and assessment of degree of resection. Performing such procedures under local anesthesia with monitored conscious sedation combined with cortical mapping can decrease the neurological morbidity for lesions in critical areas and also decreases the morbidity associated with general anesthesia; the latter combination results in shorter length of stay for patients undergoing major craniotomy for resection of intra-axial lesions.

Outpatient surgery is routine in peripheral nerve surgery, many plastic surgical and cosmetic procedures, and most arthroscopic surgeries; that is, surgeries which do not involve entry into a major body cavity or compartment such as the thorax, abdomen, and cranium. This results from the logical but to-date untested belief by patients and physicians alike that postoperative monitoring in a hospital setting with sophisticated equipment and skilled nurses and doctors is necessary after such major surgeries on vital organs within major body cavities. Since 1991, the author has routinely applied awake image-guided surgery to the majority of patients requiring craniotomy for resection of an intra-axial neoplasm, namely gliomas and metastases. $^{21}$ It has been observed that the incidence of morbidity the first night after surgery is exquisitely low and that most patients can be safely discharged the following morning. This lead the author to initiate, in late 1996, a pilot study of outpatient, or day surgery craniotomy for patients with intraaxial brain neoplasms.

\section{METHODS}

\section{Definition}

Outpatient craniotomy is defined herein as a craniotomy (ie. not bur hole), for resection (ie. not biopsy), of an intra-axial tumor in which the patient arrives at the hospital in the morning and leaves hospital without spending an overnight in hospital. Furthermore, the procedure is booked as such $a b$ initio; that is, early discharge is not decided after the fact.

\section{Patient selection}

Patients requiring awake image-guided craniotomy for resection of an intra-axial neoplasm were offered the opportunity to participate in the outpatient pilot study. Eligible patients were those living with a spouse, partner or other deemed capable of recognizing a change in the patient's neurological status. Patients with significant medical co-morbidity or significant existing neurological deficit altering their mobility were excluded. If eligible patients stated a preference to remain in hospital overnight their request was honoured. Those that accepted were educated as thoroughly as possible as to what to expect by the neurosurgeon, and preadmission work-up, including consultation with an anesthetist, arranged immediately following the office visit with the neurosurgeon.

\section{Surgical protocol}

On the morning of surgery, the patient presents to the Day Surgery Unit (DSU) at the Toronto Western Hospital at 06:30 a.m. For the vast majority in whom a frameless stereotactic navigation system was used, the patient has a gadolinium-enhanced magnetic resonance imaging (MRI) at 07:15 a.m. After the MRI, the patient is transported to the operating room and has an intravenous line placed by the anesthetist. Arterial lines, urinary catheters and central venous pressure lines are almost never used. Nasal prongs for oxygen are placed along with standard leads for electrocardiographic monitoring, pulse oximeter to measure oxygen saturation and an automatic blood pressure cuff. The patient is then positioned in the desired position (ie. lateral decubitus, supine, or sitting) and the head fixed in the Sugita head rest using pin-site local anesthesia with $2 \%$ xylocaine injection. Next the frameless navigation system is registered (Surgical Navigation Network 3.0 open platform for image-guided surgery, Surgical Navigation Specialists, Mississauga, Ontario, Canada) or, if the patient is being operated within the open magnet, a localising image is taken. Prophylactic antibiotics and, usually, steroids are administered. Anti-epileptic medications are not used routinely unless the patient is already on medication for seizures. Mannitol is not administered routinely.

Once the scalp flap has been localised, the desired area is shaved and infiltrated with $30-50 \mathrm{cc}$ of $0.25 \%$ bupivicaine with epinephrine. Sterile draping of the surgical area is done to ensure that the patient's face is free of drapes and in visual, tactile, and vocal contact with the anesthetist. A microphone is placed near the patient's mouth to facilitate communication with the surgeon. Standard craniotomy and microneurosurgical resection of the tumor is performed with the use of the frameless navigation system as needed. Prior to making the corticotomy, cortical stimulation with monopolar stimulation while testing appropriate neurological functions, is performed. During the pin placement, the infiltration of the scalp incision and the bone work, sedation is temporarily induced with short-acting intravenous agents such as propofol, midazolam, fentanyl, or remifentanil. The details of our awake craniotomy procedure have been previously reported..$^{21}$

\section{Aftercare}

At the end of surgery, which is generally before 12:00 noon, the patient is observed in the Post Anesthetic Care Unit (PACU) for four hours. If the patient remains well, he/she is then transferred to the DSU where he/she is observed to walk and given a small snack of crackers and clear liquids. The neurosurgeon reviews the patient at around 5:00 p.m. and discharges him/her if he/she is feeling well and is not neurologically worse than pre-operatively. The patient and loved one(s) are reminded of what negative signs to be aware of and 

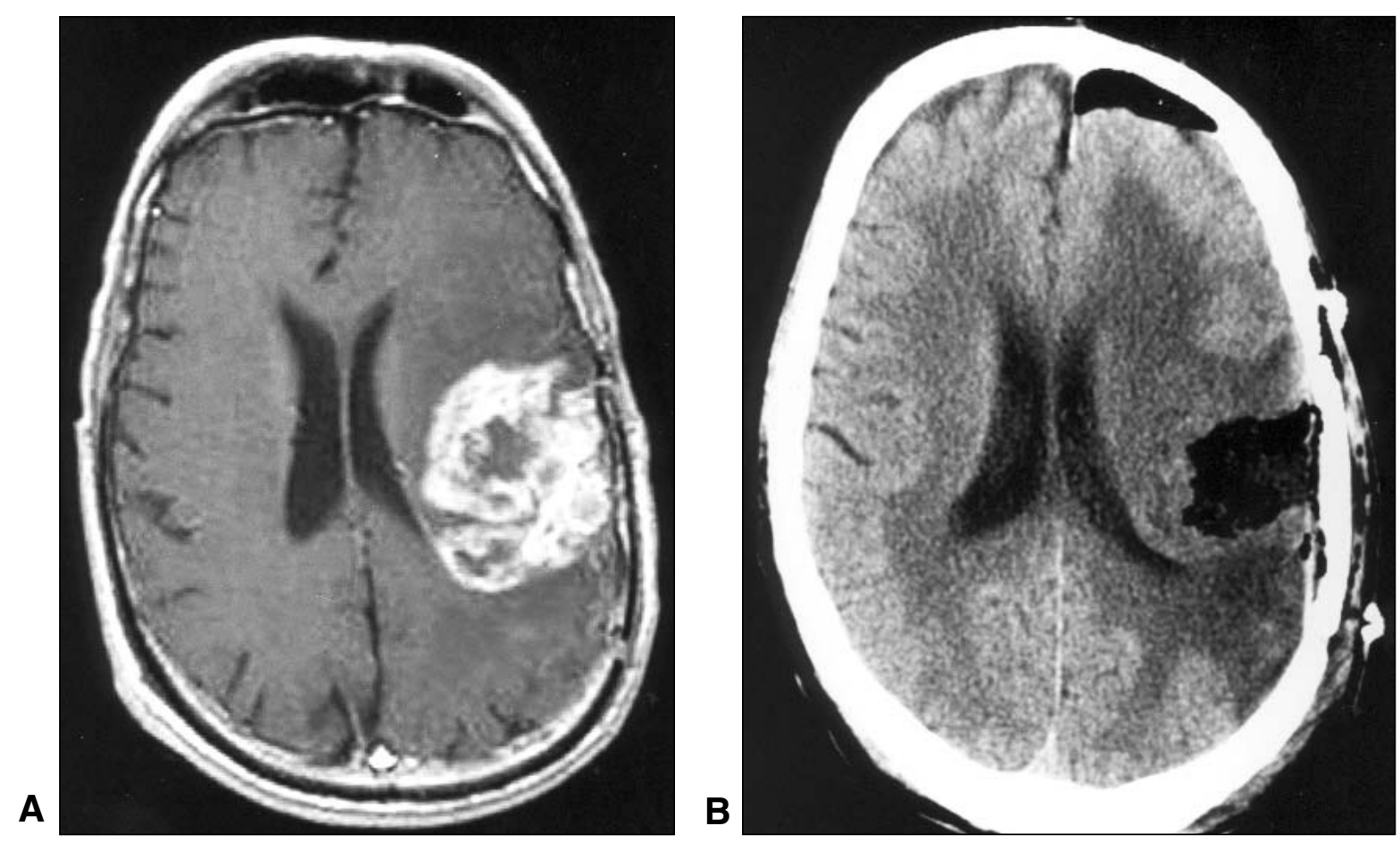

Figure 1: A. Axial gadolinium-enhanced T1-weighted MRI of a 56-year-old man with recurrent glioblastoma before awake image-guided craniotomy. The patient had mild dysphasia and mild right hemiparesis.

B. Unenhanced computed tomographic scan done three hours after aggressive subtotal resection of tumor. Patient was discharged home to his family's care several hours later with no complications and went on to receive adjuvant outpatient experimental chemotherapy

given the surgeon's pager number and home phone number. A Home Care nurse visits the patient at home at 11:00 p.m. that evening and at 08:00 a.m. the following morning. The patient is reviewed in the surgeon's office in five to seven days to examine the wound, perform a neurological assessment, discuss the final pathology with the patient, and arrange for further therapy such as radiation and medical oncology appointments and further imaging. The patient is also asked about his/her overall satisfaction with the care, and specifically with the fact that he/she was discharged on the same day as the brain surgery as opposed to remaining in hospital at least one night.

\section{RESUlts}

During the 48 month period ending December 2000, the author performed 245 image-guided awake craniotomies with cortical mapping and of those, 46 patients were entered into the study. The pathology in the 46 intent-to-treat group was highgrade glioma in 11 , low-grade glioma in 8 , metastasis in 21 , and miscellaneous in 6 . There were 21 males and 25 females. In 39 cases the operation was the patient's first craniotomy and seven operations were a repeat craniotomy. There were 25 left sided and 21 right sided tumors. The median Karnofsky Performance Score was 80 and the range 60-100. Tumors of various location, size, and associated mass effect were represented in this study. In other words, all of the tumors were not small, superficial tumors in noneloquent locations (Figures 1,2). Details of the patients, tumors, and surgery are summarized in the Table.
Four patients were converted to inpatients because of: (1) immediate postoperative hemiparesis (which ultimately resolved) in a patient with a large low-grade glioma; (2) air embolus (with no permanent sequelae) in a patient with a motor cortex metastasis from bronchogenic carcinoma; (3) intraoperative grand mal seizure requiring conversion to general anesthetic in a patient with an oligodendroglioma, with no permanent sequelae; and (4) request by a family member who was not present at the initial pre-operative discussions with a patient with a temporal low-grade astrocytoma. One other patient, an elderly gentleman with a frontal metastasis who was discharged as per the protocol returned to the hospital later the same evening because of headache and was admitted for observation overnight. Therefore 41/46 (89\%) patients successfully completed the outpatient craniotomy protocol. There were five complications in the intent-to-treat group (10.9\%): (1) the one case of postoperative hemiparesis; (2) the one case of intra-operative air embolus; (3) the one case of intraoperative grand mal seizure; (4) one case of mild increase of a pre-operative hemiparesis; and (5) a case of delayed wound healing in an elderly patient operated for a metastasis from bronchogenic carcinoma. There were two complications in the 41 patients who successfully completed the outpatient protocol (4.9\%).

No patient was disadvantaged by participating in the study; that is no patient suffered neurological or other deterioration at home which altered their quality or quantity of life. At follow-up visit the following week, all patients were asked about their 

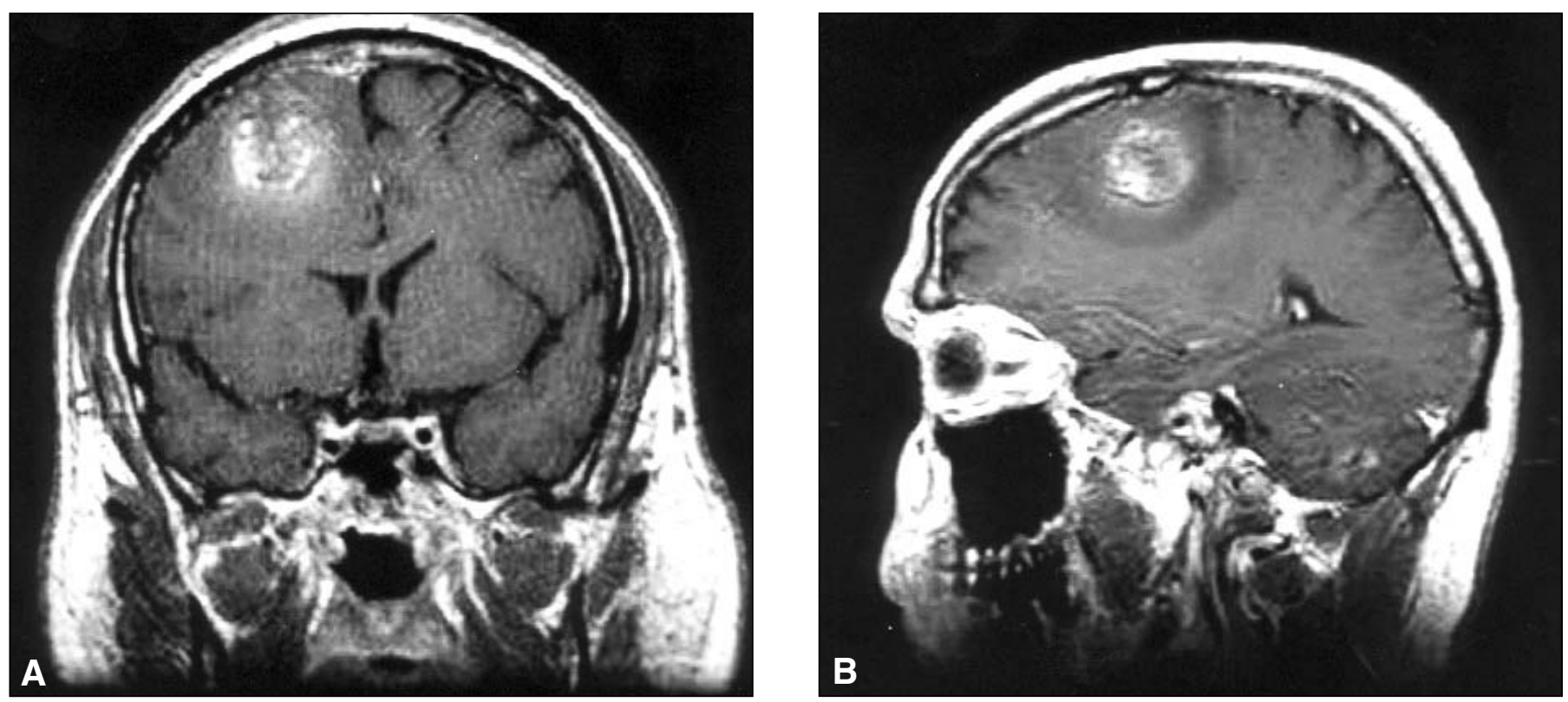

Figure 2: Gadolinium-enhanced T1-weighted MRI in the coronal (A) and sagittal (B) planes showing right frontal subcortical tumor with edema and mass effect in a 28-year-old man who underwent outpatient craniotomy for metastatic germ cell tumor.

satisfaction with the outpatient craniotomy procedure and no patient verbalized any discontent to the surgeon, but detailed self-report confidential satisfaction surveys were not administered.

\section{Discussion}

Patients with anaplastic gliomas, and those with brain metastases have a median survival measurable in months to years. Less time spent within the walls of a hospital may possibly translate into improved quality of life, at least in the short term. Furthermore, the fear and anxiety associated with brain tumor surgery, especially when this is added to the knowledge by the patient that they likely harbour a malignant intracranial process, may well be diminished when they learn that the tumor resection can be done as an outpatient procedure. It is also possible that nosocomial complications such as colonization with resistant microorganisms and thromboembolic events would be diminished by immediate mobilisation and discharge of patients who already have a disease process and a therapeutic intervention which predispose them to these morbidities. Shorter time spent in hospital might also reduce the chance of an adverse event caused by an error, a phenomenon becoming more widely recognized as a realtively common occurrence in hospitals. ${ }^{22}$ These are the potential advantages to the patient of outpatient craniotomy for a malignant intra-axial tumor. Other system advantages include the reduction in resource utilisation which can translate into financial savings for the health care system and liberation of resources for those patients who truly need them. Regarding the workload to surgeons, residents, family doctors, and local emergency rooms, it is estimated that there would be no significant change due to outpatient craniotomy, although these aspects were only informally tracked during this pilot study.
Table: Characteristics of the 46 patients

Characteristic Value

Sex (number of patients)

$\begin{array}{ll}\text { Male } & 21 \\ \text { Female } & 25\end{array}$

Age (years)

$\begin{array}{lr}\text { Mean } & 52 \\ \text { Range } & 18-78\end{array}$

Side of tumor

Left 25

Right 21

Location of tumor

Frontal 27

Parietal 12

Temporal 6

Occipital 1

Surgery

First operation $\quad 39$

Repeat craniotomy 7

Pathology

High-grade glioma $\quad 11$

Low-grade glioma 8

Metastasis 21

Other 6

Extent of resection

Gross total 27

Subtotal 14

Partial 5 
Performing brain tumor resection as an outpatient may appear to be a dangerous, perhaps even cavalier endeavour. However, extensive experience by the author with awake craniotomy has suggested its feasibility and the current pilot study provides further supportive evidence that it is a safe and effective treatment option in selected patients. Awake craniotomy is welltolerated by patients ${ }^{23}$ and the combination of functional guidance with anatomic navigation allows for less invasive, shorter surgeries with low neurological morbidity and low medical morbidity. These factors combine to allow for early discharge and in the current feasibility study, truly outpatient surgery.

The major concern to neurosurgeons and patients undergoing outpatient craniotomy would be missing the occurrence of a postoperative hematoma which is a well-recognized complication of tumor surgery. However, in a large series of 1,427 elective craniotomies, all postoperative hematomas presented either before six hours or after 24 hours postoperatively. ${ }^{24}$ If the patient was discharged the following morning after surgery, which is becoming more commonplace and has been the author's practice for several years, he/she would still not have been within the hospital for observation after 24 hours. All patients developing clinically relevant early hematomas would have this complication recognized while they were still in the PACU or DSU and appropriate corrective measures would be taken, including admission for either observation or surgical intervention.

It is not advised that every surgeon adopt this procedure nor is it suggested that this should represent the standard of surgical care for a patient with an intra-axial tumor. However, this study has demonstrated that outpatient craniotomy is a feasible option for the surgical management of selected patients with a survivallimiting diagnosis. It is also not suggested that this procedure would have a significant impact on patients' overall outcome such as length of survival and overall quality of life. This approach may represent an important step in the minimallyinvasive "revolution" of modern surgery, facilitating a health care system which is user-friendly and more efficient without compromising patient care. Concerns for patient safety and for self-protection against potential medicolegal consequences may limit the widespread adoption of this procedure by most neurosurgeons. Future studies including detailed satisfaction surveys and cost analysis are planned to establish the overall efficacy of outpatient craniotomy, likely in the setting of a randomized study in which the other "arm" would be patients assigned to spend an overnight in hospital.

\section{REFERENCES}

1. Canin-Endres J, Salky B, Gattorno F, Edye M. Laparoscopically assisted intestinal resection in 88 patients with Crohn's disease. Surg Endosc 1999;13:595-599.

2. Poole GH, Yellapu S. Acute laparoscopic cholecystectomy. A case controlled study. Surg Endosc 2000;14:106-109.

3. Kirkley A, Griffin S, Richards C, Miniaci, Mohtadi N. Prospective randomized clinical trial comparing the effectiveness of immediate arthroscopic stabilization versus immobilization and rehabilitation in first traumatic dislocations of the shoulder. Arthroscopy 1999;15:507-514.

4. Donovan JL, Peters TJ, Neal DE, et al. A randomized trial comparing transurethral resection of the prostate, laser therapy and conservative treatment of men with symptoms associated with benign prostatic enlargement: the ClasP study. J Urol 2000;164:65-70.

5. Canto JG, Every NR, Magid DJ, et al. The volume of primary angioplasty procedures and survival after acute myocardial infarction. N Engl J Med 2000;342:1573-1580.

6. Faria MA, Tindall GT. Transsphenoidal microsurgery for prolactinsecreting pituitary adenomas. J Neurosurg 1982;56:33-43.

7. Yaniv E, Rappaport ZH. Endoscopic transseptal transsphenoidal surgery for pituitary tumors. Neurosurgery 1997;40:944-946.

8. Vanaclocha V, Saiz-Sapena N, Panta F. Uniportal endoscopic superior thoracic sympathectomy. Neurosurgery 2000;46:924928.

9. Fukuhara T, Vorster SJ, Luciano MG. Risk factors for failure of endoscopic third ventriculostomy for obstructive hydrocephalus. Neurosurgery 2000;46:1100-1111.

10. Katkhouda N, Campos GM, Mavor E, et al. Is laparoscopic approach to lumbar spine fusion worthwhile? Am J Surg 1999; 178:458-461.

11. Hermantin FU, Peters T, Quartararo L, Kambin P. A prospective, randomized study comparing the results of open discectomy with those of video-assisted arthroscopic microdiscectomy. J Bone Joint Surg 1999;81-A:958-965.

12. Albuquerque FC, Teitelbaum GP, Lavine SD, Larsen DW, Giannotta SL. Balloon-protected carotid angioplasty. Neurosurgery 2000; 46:918-923.

13. Vanninen R, Koivisto T, Saari T, Hernesniemi J, Vapalahati M. Ruptured intracranial aneurysms: acute endovascular treatment with electrolytically detachable coils - a prospective randomized study. Radiology 1999;211:325-336.

14. Qureshi AI, Luft AR, Sharma M, Guterman LR, Hopkins LN. Prevention and treatment of thromboembolic and ischemic complications associated with endovascular procedures: part II Clinical aspects and recommendations. Neurosurgery 2000;46: 1360-1376.

15. Bernstein M, Parrent AG. Complications of CT-guided stereotactic biopsy of intra-axial brain lesions. J Neurosurg 1994;81:165-168.

16. Fine J, Duff J, Chen R, et al. Long-term follow-up of unilateral pallidotomy in advanced Parkinson's disease. N Engl J Med 2000;342:1708-1714.

17. Bernstein M, Laperriere N, Glen J, et al. Brachytherapy for recurrent malignant astrocytoma. Int $\mathrm{J}$ Rad Onc Biol Phys 1994;30:1213-1217.

18. Prasad D, Steiner M, Steiner L. Gamma surgery for vestibular schwannoma. J Neurosurg 2000;92:745-759.

19. Golfinos JG, Fitzpatrick BC, Smith LR, Spetzler RF. Clinical use of a frameless stereotactic arm: results of 325 cases. J Neurosurg 1995;83:197-205.

20. Bernstein M, Al-Anazi AR, Kucharczyk W, et al. Brain tumor surgery with the Toronto open magnetic resonance imaging system: preliminary results for 36 patients and analysis of advantages, disadvantages, and future prospects. Neurosurgery 2000;46:900-909.

21. Taylor MD, Bernstein M. Awake craniotomy with brain mapping as the routine approach to treating patients with supratentorial intraaxial tumors: a prospective trial of 200 cases. J Neurosurg 1999;90:35-41.

22. Brennan TA, Leape LL, Laird NM, et al. Incidence of adverse events and negligence in hospitalized patients. Results of the Harvard Medical Practice Study I. N Engl J Med 1991;324:370376.

23. Danks RA, Rogers M, Aglio LS, Gugino LD, Black PM. Patient tolerance of craniotomy performed with the patient under local anesthesia with monitored conscious sedation. Neurosurgery 1998;42:28-34.

24. Taylor WAS, Thomas NWM, Wellings JA, Bell BA. Timing of postoperative intracranial hematoma development and implications for the best use of neurosurgical intensive care. J Neurosurg 1995;82:48-50. 